\title{
Navier-Stokes Analysis of a High Wing Transport High-Lift Configuration With Externally Blown Flaps
}

Jeffrey P. Slotnick, Michael Y. An, Stephen J. Mysko, and David T. Yeh The Boeing Company

Stuart E. Rogers and Karlin Roth

NASA Ames Research Center

M. David Baker and S. Nash

MCAT, Inc.

\section{$\underline{\text { Abstract }}$}

Insights and lessons learned from the aerodynamic analysis of the High Wing Transport (HWT) high-lift configuration are presented. Three-dimensional Navier-Stokes CFD simulations using the OVERFLOW flow solver are compared with high Reynolds test data obtained in the NASA Ames 12 Foot Pressure Wind Tunnel (PWT) facility. Computational analysis of the baseline HWT high-lift configuration with and without Externally Blown Flap (EBF) jet effects is highlighted. Several additional aerodynamic investigations, such as nacelle strake effectiveness and wake vortex studies, are presented. Technical capabilities and shortcomings of the computational method are discussed and summarized.

\section{Introduction}

To reduce the costs associated with the design, development, and manufacturing of modern transport aircraft, major improvements in both aircraft development cycle (time-tomarket) and affordability (flyaway cost) must be achieved in order to make new concepts commercially viable. The major driver in reducing aircraft development cycle time and total system cost is the reduction of aerodynamic design cycle time.

To enable these improvements, significant efforts to utilize CFD for high-lift system design are underway in order to greatly reduce the amount of wind tunnel testing required in the design process. Emerging Navier-Stokes methods that model the viscous-dominated flow physics associated with advanced transport aircraft aerodynamics have the potential to reduce aerodynamic design cycle time. However, to be successful, CFD methods must be easy to use, fast, and accurate.

High-lift analysis is particularly challenging, and remains the pacing item in reducing aerodynamic cycle time mainly due to geometric complexity, and the wide range of flow phenomena typically present in high-lift flowfields. High-lift aerodynamics is extremely complex, and is dominated by viscous flow phenomena, such as separation, shock-boundary layer interactions, and multiple element merging confluent wakes. A significant body of work has been performed in $2 \mathrm{D}$ to characterize high-lift flow physics ${ }^{1,2}$. However, since high lift flows are inherently three-dimensional, testing and simulation of complete transport configurations are required to accurately predict high lift system performance.

Computing the three-dimensional subsonic flow over a complete transport high lift configuration is one of the most difficult challenges of modern $\mathrm{CFD}^{3}$. Inclusion of engine jet impingement effects in the simulation of EBF-powered high lift systems remains a pacing item for emerging CFD technology. Under the Integrated Wing Design (IWD) element of the NASA 
Advanced Subsonic Technology (AST) program, a significant effort was focused on developing the CFD software tools required to do production level CFD analysis of 3D high-lift systems on complete transport configurations. As a result of these efforts, the CFD analysis time associated with generating a CFD simulation from geometry to final solution on a complete high-lift transport configuration was reduced by an order of magnitude. This technology was used extensively to analyze the aerodynamic performance of the HWT EBF-powered high-lift configuration.

This paper discusses some of the successes and shortcomings involved in the numerical simulation of the complete HWT configuration high-lift configuration using modern NavierStokes methods. Outstanding issues include facility wall interference effects, jet modeling adequacy, and grid density effects.

\section{Experimental Data and Computational Analysis}

Under the AST-IWD program, extensive high Reynolds number powered test data was obtained for the semispan HWT model in the NASA Ames Research Center's 12 Foot Pressure Wind Tunnel (PWT) facility. Turbine Powered Simulator (TPS) units were used to simulate the engine exhaust flow. Data collected included standard force and moments, and a vast array of surface pressure transducer measurements on the fuselage, the main wing, the high lift elements (slat, vane, and flap), and winglet. Also, flowfield pressure and temperature measurements were obtained using the Boeing Quantitative Wake Survey System (QWSS). An additional test entry, designed for TPS calibration, was used to collect jet flow QWSS flowfield data.

The Chimera overset grid method was employed to discretize the HWT surface and volume domain. The OVERFLOW Navier-Stokes flow solver, with multigrid convergence acceleration, was used to solve the viscous HWT high-lift flowfield. All computations are fully turbulent, and employ the Spalart-Allmaras one-equation turbulence model. The computational model of the baseline HWT high-lift configuration at forty degrees flap deflection installed in a computational model of the NASA Ames 12 Foot PWT facility is shown in Fig. 1. The HWT overset grid system is composed of 153 grids and 35.2 million grid points. The grid model contains all geometry detail except the empennage, slat brackets, and vane brackets.

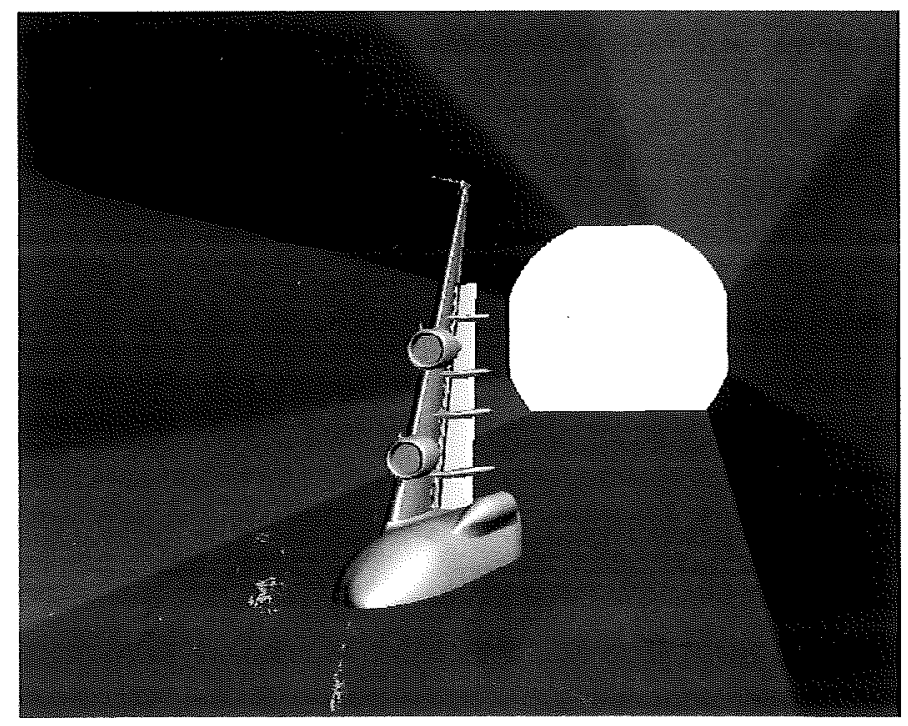

Figure 1. HWT computational geometry model. 


\section{Baseline HWT High-Lift Configuration Results}

To evaluate the capability of the OVERFLOW code to accurately predict the aerodynamic performance of the HWT high-lift configuration, several simulations with and without EBF power effects at a range of angle-of-attack were computed. Comparison of the lift curve slope between experimental data and CFD simulations is shown in Fig. 2. This data is for the baseline vane-flap configuration deflected 40 degrees, with wind tunnel walls modeled in both unpowered $\left(c_{\mu}=0.03\right)$ and powered $\left(c_{\mu}=0.5\right)$ modes, where $c_{\mu}$ is the engine thrust coefficient. The difference between the experimental and CFD data at each CFD flow condition is given in percent. Also, the linear portion of each experimental lift curve has been extrapolated and is plotted with black, dashed lines. In general, CFD results compare very well with unpowered test data up to Clmax. However, the powered computational results differ from the experimental results. The primary reasons for these differences are thought to come from at least two different sources. The first of these is the inaccuracies in the computation of the engine jets. Evidence of this is presented below. The second factor is the effect of the wind tunnel test section. In this flowfield, the EBF jet exhaust is deflected down due to the presence of the flap, but is forced to bend downstream (upward) prematurely at the wall. As a result, the large wall interference effect effectively pushes the jet up closer to the flap surface. With more of the jet impinging on the flap at higher angles-of-attack in the experiment than is experienced in free-air, the lift increases nonlinearly up to stall. This explains the non-linear bending of the powered lift curve at the high, pre-stall angles of attack. The computational geometry does include an inviscid, simplified model of the wind tunnel test section, but it neglects the effects of viscosity of the test section splitter plate and semispan mounting apparatus, and the divergence of the wind tunnel walls. The current CFD results predict the trend of the non-linear lift curve, but not to the same magnitude as found in the experimental results.

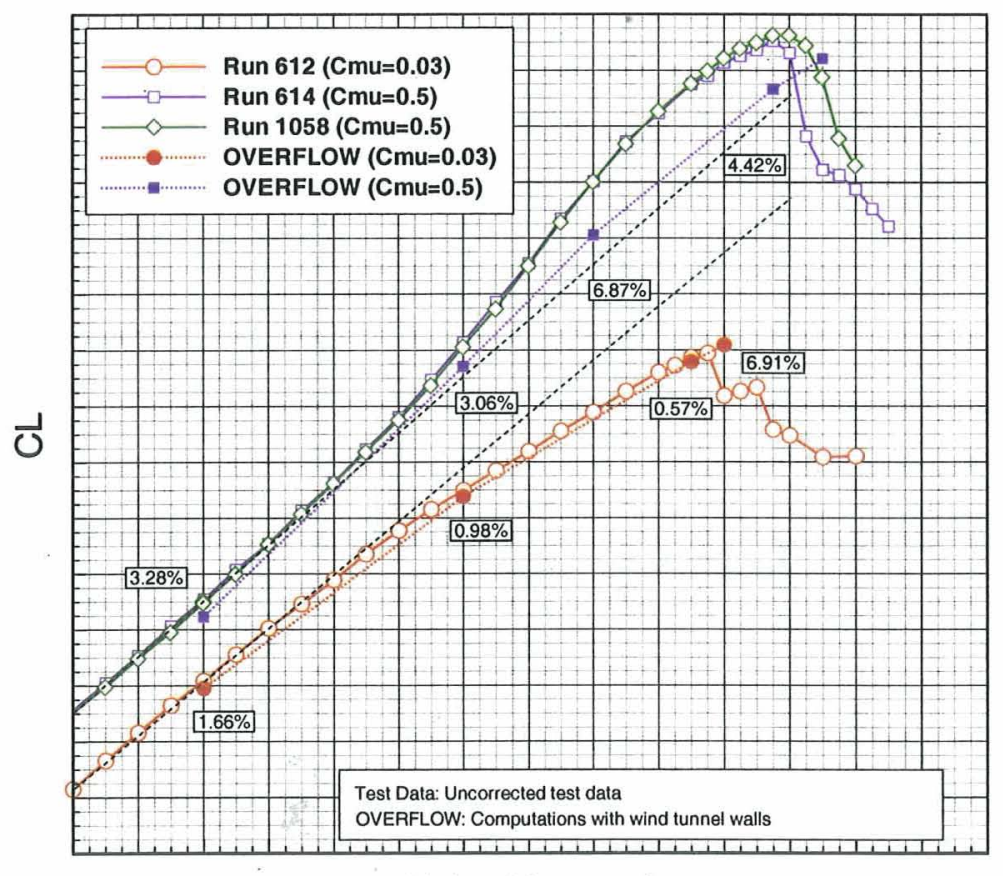

Alpha (degrees)

Figure 2. Comparison of total configuration lift. 
Surface pressures also show good agreement between CFD and test data for the baseline HWT configuration at zero degrees angle-of-attack. Figure 3 shows the slat, main wing, vane, and flap element surface pressure comparisons between CFD and test data at five spanwise rows of pressure taps. For the unpowered case, pressure comparisons between CFD and test data at the pressure rows that bracket the engine jet streams are excellent. For the powered case, however, OVERFLOW does not predict the large pressure rise due to the jet exhaust impingement on the flap at $34 \%$ and $60 \%$ span. These differences are attributed not only to wall interference effects, but also lack of adequate CFD jet modeling.
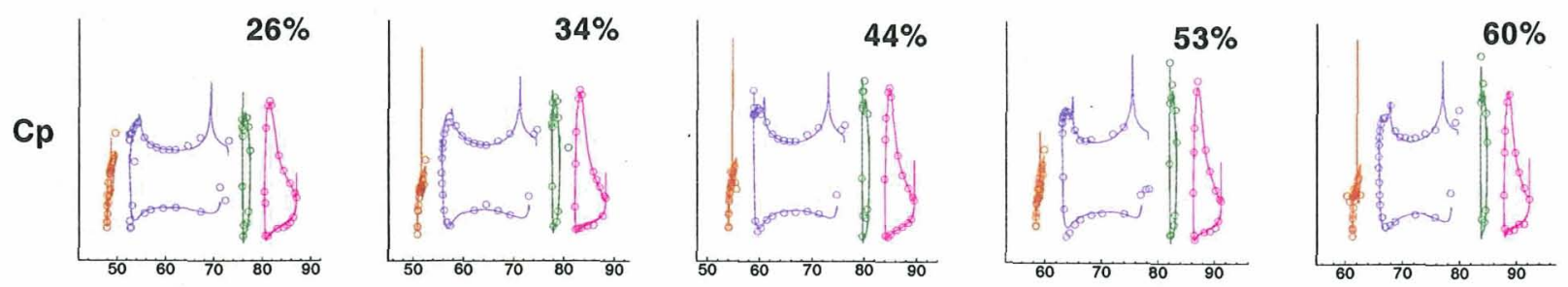

$\mathrm{X}$ Location

(a) Power-off
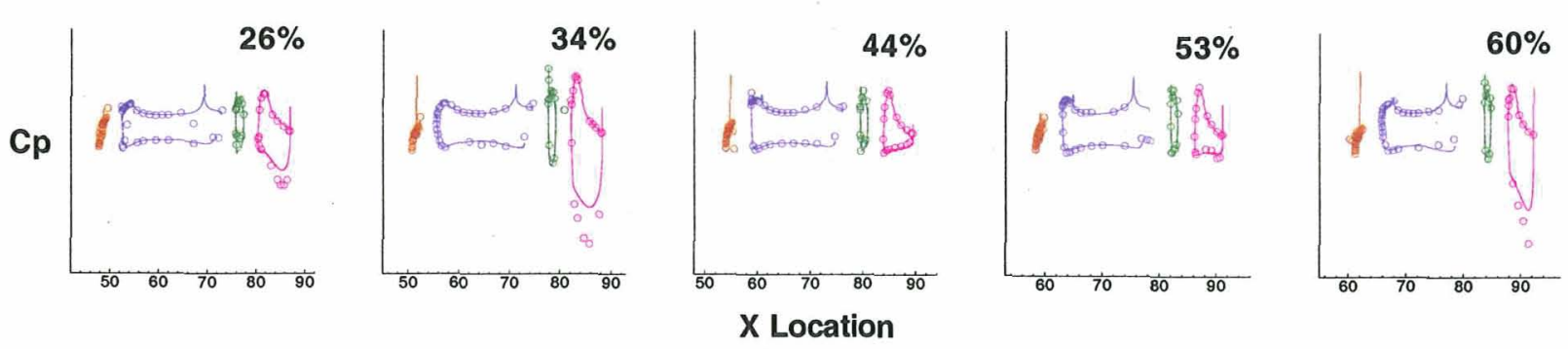

(b) Power-on

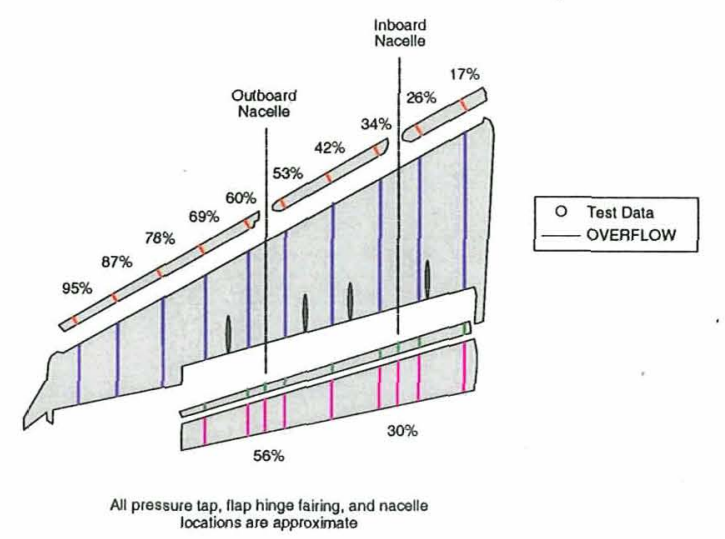

Figure 3. Comparison of surface pressures, $\alpha=0$ degrees.

To illustrate the jet modeling capability of the OVERFLOW code, comparisons of QWSS jet flowfield data collected during the isolated TPS calibration experiment with CFD results are presented. Fig. 4 shows the computational model and representative OVERFLOW Mach contours for the TPS calibration configuration. The CFD model contains 2.6 million points in 32 grids. Also shown are the three streamwise plane stations where flowfield data was collected. Fig. 5 shows the comparison of test data (top) with OVERFLOW computational results (bottom) using the S-A turbulence model. The experimental data clearly shows an asymmetric distortion of the jet at all three QWSS stations. This is likely caused by flow swirl in the TPS unit, and 
perhaps influenced by the presence of total pressure rakes inside the fan and core ducts. For the OVERFLOW simulations, jet boundary conditions are defined as average, radially-constant flow without swirl. Even with this approximation, the basic magnitude of the jet flow is modeled well with CFD. However, in these cases using the 1-equation S-A turbulence model, the jet stream mixing and spreading are underpredicted. Since OVERFLOW does not accurately predict the proper spreading of the jet at the HWT flap (very near station 3), there is less jet impingement on the flap, resulting in lower total configuration lift relative to test data.
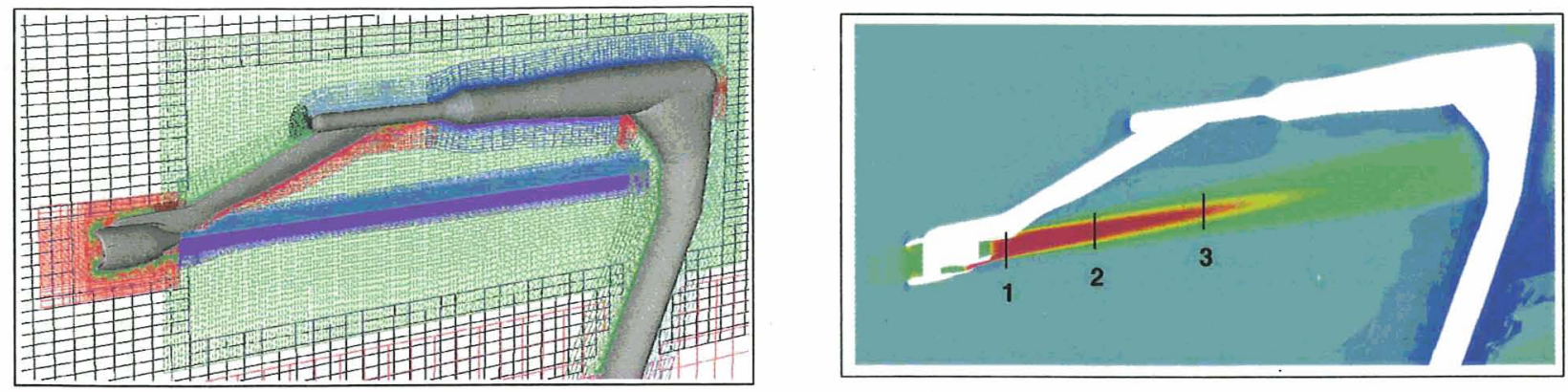

Figure 4. TPS calibration model grid system and representative OVERFLOW solution.

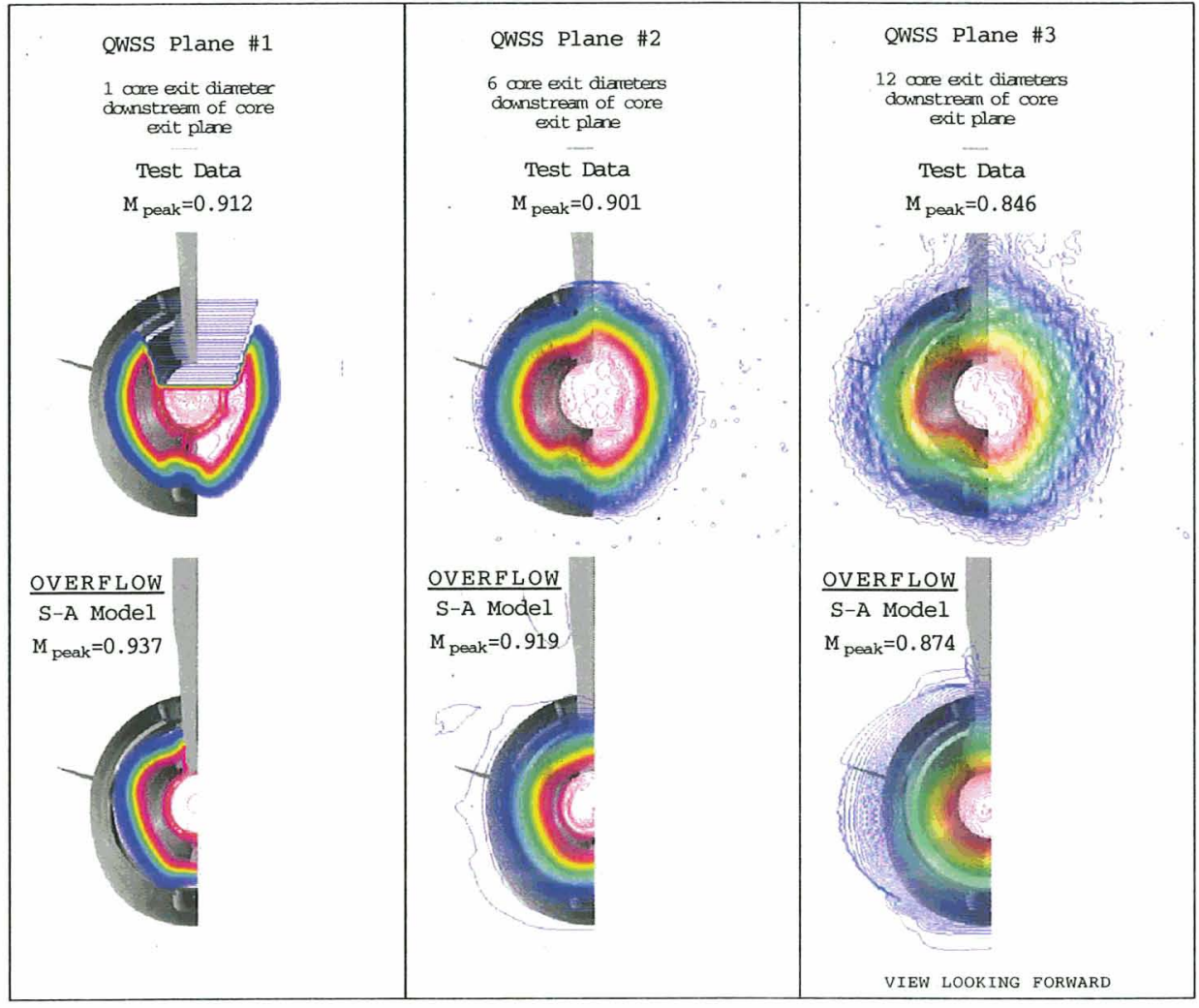

Figure 5. QWSS Mach number comparisons.

\section{Nacelle Strake Effectiveness Studies}

In addition to the analysis of the baseline HWT high-lift flowfield, CFD has been used with much success to study specific, detailed aspects of HWT aerodynamic performance. Increased understanding of nacelle strake effectiveness on maximum lift, for example, is critical to properly optimize strake size and location. Moreover, determining the computational 
requirements to predict these strake effects is equally as important. For these purposes, the ability of Navier-Stokes CFD methods to capture nacelle strake effects was evaluated.

OVERFLOW solutions at an angle-of-attack near stall were obtained both with all strakes on and with all strakes off. Nacelle strakes are designed to increase Clmax through two flow mechanisms. First, nacelle strakes relieve the adverse flow effects at the wing-pylon intersection by reducing nacelle upwash. Fig. 6 clears shows the reduction of upwash with strakes on. As a result, cross-flow separation on the nacelle near the pylon juncture is reduced (Fig. 7).

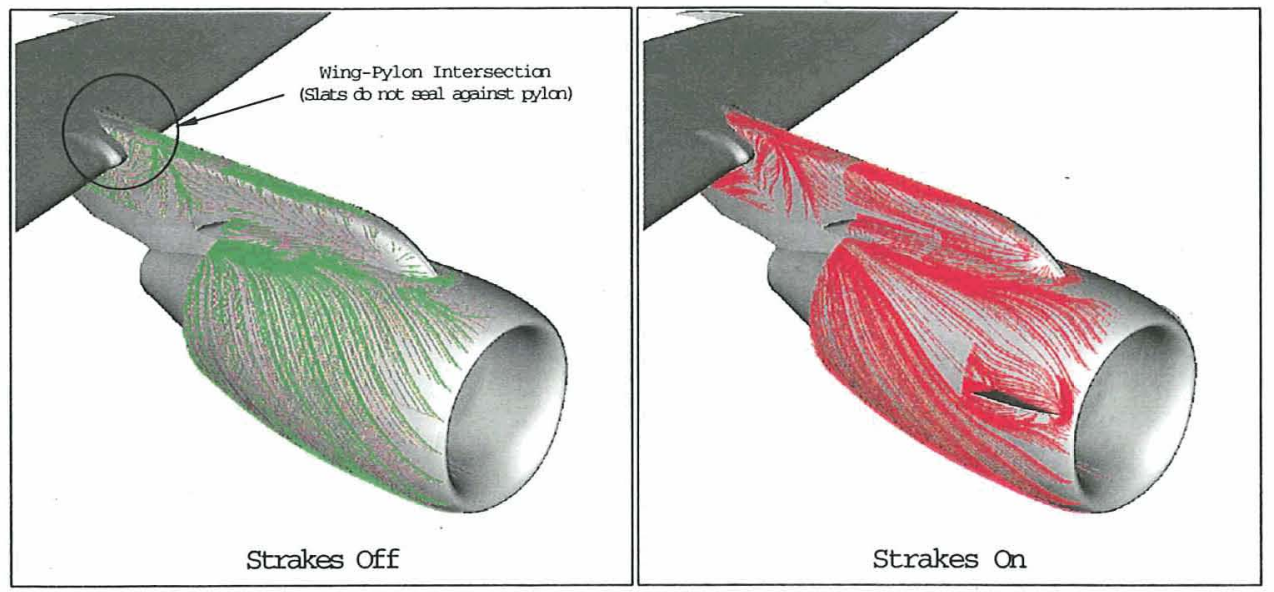

Figure 6. Nacelle surface oilflow.

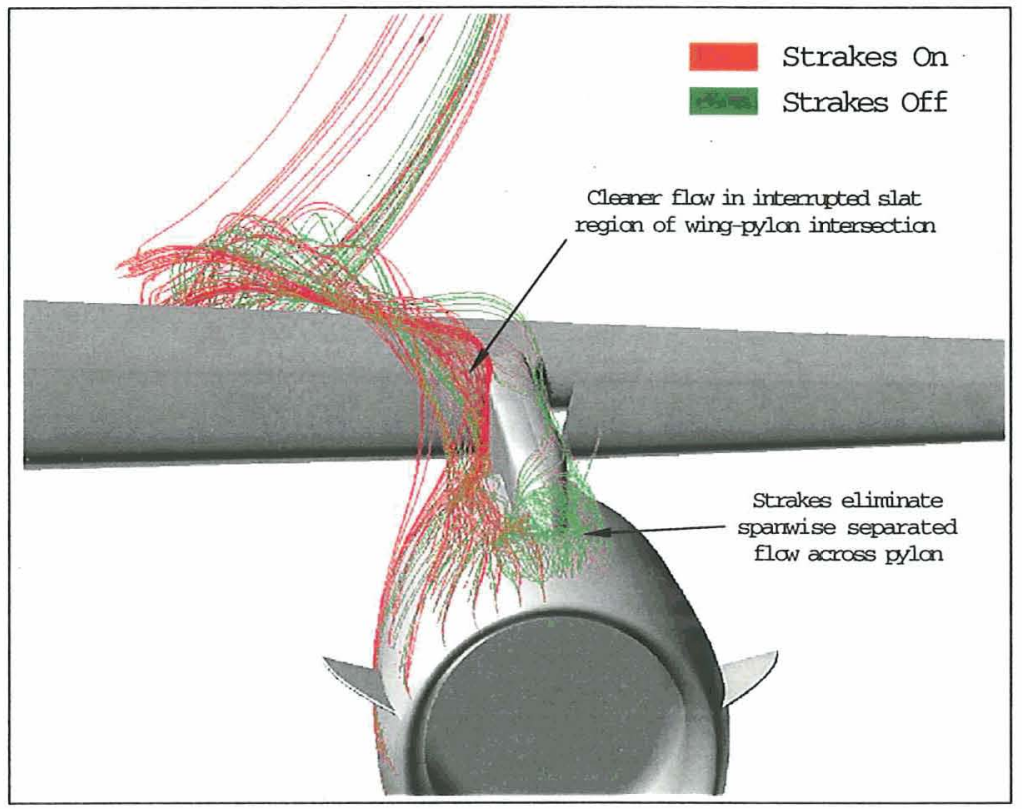

Figure 7. OVERFLOW nacelle strake particle traces.

Second, the presence of the strake vortices provides downwash on the wing upper surface that serves to energize the boundary layer. Fig. 8 depicts total pressure contours at two streamwise locations on the wing aft of the outboard nacelle/pylon. For the strake-off configuration, the flow tends pull away from the surface. The strake vortices provide downwash that effectively eliminates this flow behavior. As a result, the health of the boundary layer is improved, and flow 
separation is delayed. In general, OVERFLOW CFD analysis has greatly increased our understanding of the flow physics mechanism associated with how the presence of nacelle strakes increases configuration maximum lift.

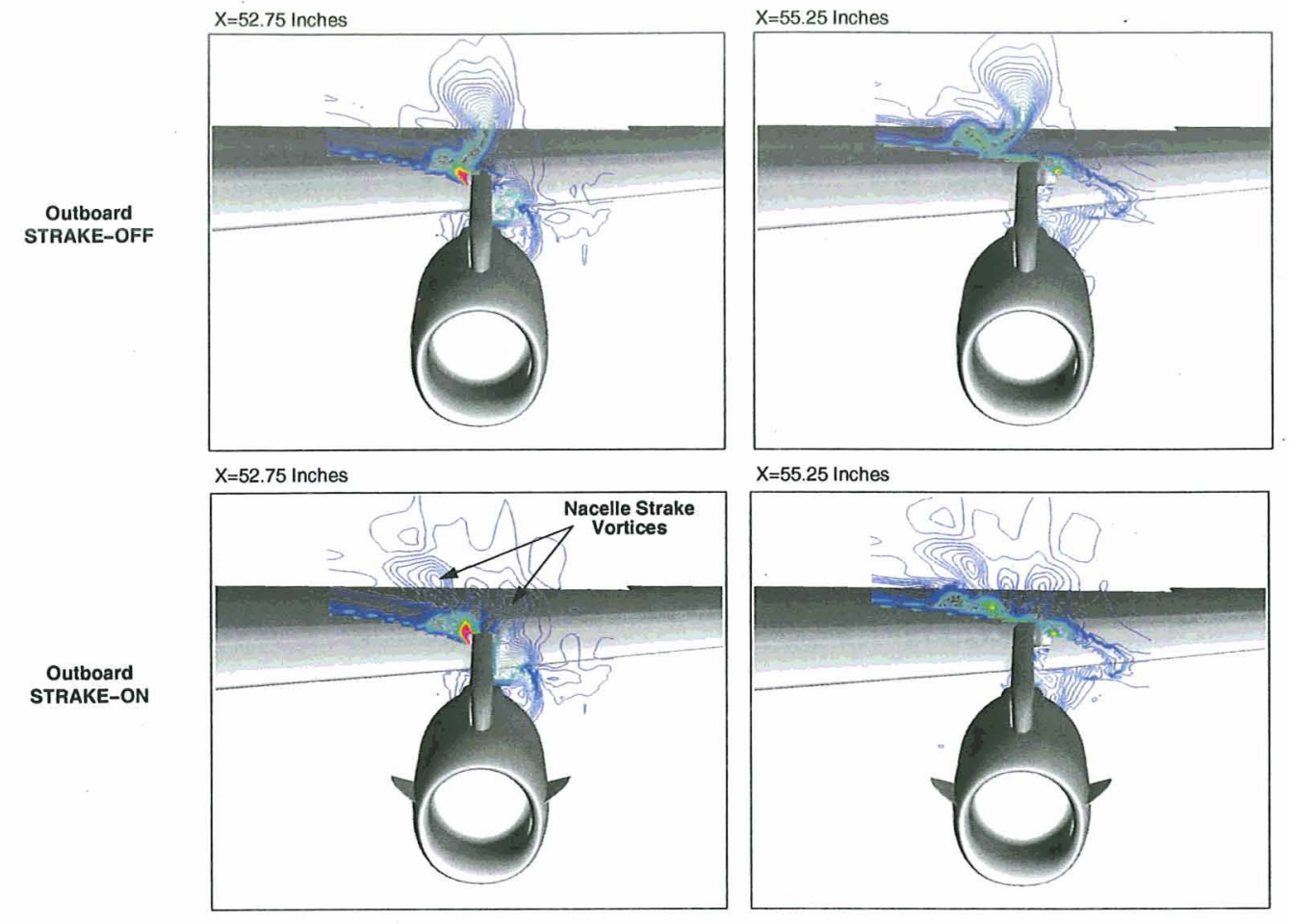

\section{Wake Vortex Studies}

To evaluate the capability of the OVERFLOW code to predict near-field wake vortex structure (to ultimately assess vortex alleviation concepts), several CFD simulations for a variety of flap configurations were obtained, and compared with QWSS flowfield data collected during the HWT model test. The constant streamwise QWSS measurement plane is located approximately one tip chord downstream of the wing trailing edge. Fig. 9 shows the Mach number contour comparison for the baseline 40 degree vane-flap configuration at a representative approach power condition.

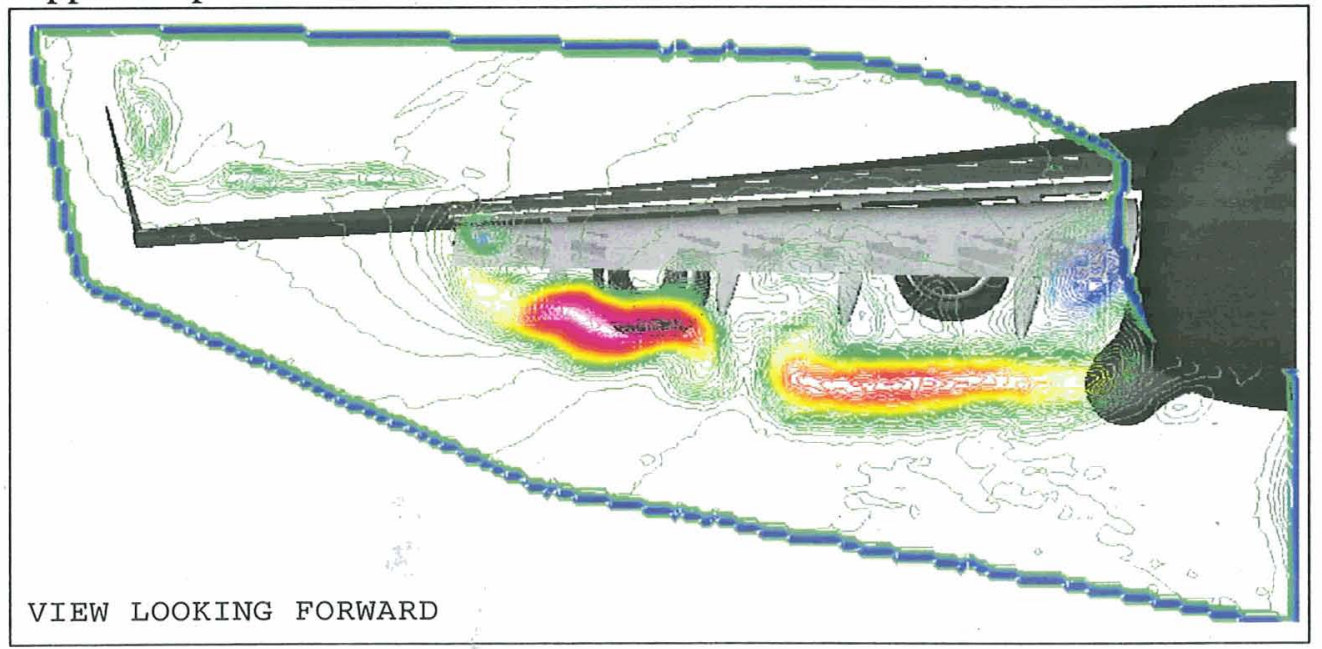

(a) Test data. 


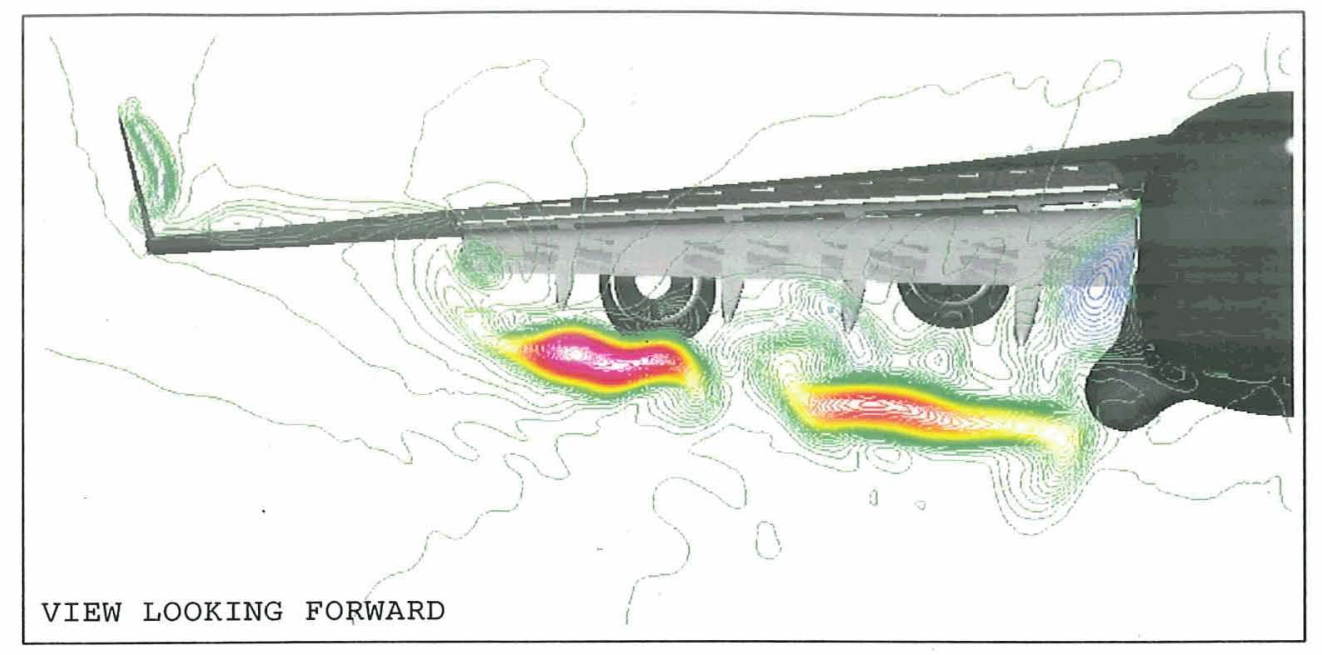

(b) Computation result.

Figure 9. Comparison of Mach number contours in QWSS wake vortex survey plane.

In general, the CFD results and experimental data agree quite well. The distortion of both the inboard and outboard engine jet exhaust downstream of the flap is well predicted by OVERFLOW. However, due to a significantly greater wall interference effect seen in the test data relative to the CFD simulations, the overall experimental wake flowfield is shifted up vertically, so that the jet exhaust stream is closer to the wing. Subsequently, OVERFLOW has been used to analyze the wake vortex structure of several alternate flap configurations. These alternate flap configurations are designed to minimize wake vortex strength.

\section{$\underline{\text { Conclusions }}$}

The OVERFLOW code has been applied to the aerodynamic analysis of the complete HWT high-lift configuration. Power-off simulations compare well with experimental data. However, power-on simulations underpredict the total configuration lift relative to the test data, due to wind tunnel wall interference effects and inadequate turbulence modeling for jet flows. Even with these shortcomings, OVERFLOW CFD results have been successfully used to gain valuable insights into the flow physics associated with nacelle strake effectiveness and wake vortex formation.

\section{$\underline{\text { References }}$}

[1] Rogers. S. E., "Progress in High-Lift Aerodynamic Calculations," AIAA Paper 93-0194, Jan. 1993. Also published in the Journal of Aircraft, Vol 31, No. 6, Nov. 1994, pp 1244 1251.

[2] Cao, H. V. and Kusunose, K., "Grid Generation and Navier-Stokes Analysis for MultiElement Airfoils,” AIAA Paper 94-0748, January, 1994.

[3] Cao, H. V., Su, T. Y., and Rogers, S. E., "Navier-Stokes Analysis of a 747 High Lift Configuration," AIAA Paper 98-2623, July 1998. 